\title{
Measurement of Escaping Ions in the Fermilab Antiproton Accumulator
}

\author{
P. Zhou, P. L. Colestock, K. Junck and C. A. Crawford \\ Fermi National Accelerator Laboratory* \\ P.O. Box 500, Batavia, IL 60510
}

\section{Abstract}

Positively charged ions trapped in the negatively charged beam of the Fermilab antiproton accumulator pose a limit to beam stability and density. To better understand the dynamics and the consequences of the beam-ion interaction, we have built and installed a low energy ion detector and energy analyzer in the Fermilab accumulator. This analyzer is capable of energy analysis of the escaping ions using a probe with energy retarding grids and may also be scanned in the pitch angle of the escaping ions. Measurements have been made in both longitudinal and transverse planes under a variety of machine operating conditions. The experimental measurement results will be presented together with attempts to model the ion dynamics and explain observations.

\section{Introduction}

Ion trapping in accelerators with negatively charged beams occurs when ions created through Coulomb collisions with beam particles are not energetic enough to escape the beam electric field. This is a very complex process as it involves the constant creation, interaction with the beam and the background gas molecules, and escape of the ions. A direct measurement of the ions would provide some of the information needed to understand the interaction process.

Fermilab Antiproton Accumulator is a DC storage ring of antiprotons where ion trapping is a major problem if uncorrected. The average vacuum in the accumulator is $2 \times 10^{-10}$ torr. Calculations using Bethe theory with experimentally measured constants [1] and by clearing current measurements both show a neutralization time, in which beam will completely neutralize its own charge, of about 2 seconds. While the incoherent effects due to trapped ions are small and can be ignored when the ion clearing system maintains the overall neutralization level to few percent, the coherent interaction between the beam and trappedions are still causing noticeable effects on the beam quality. An ion detector and energy analyzer, IDEA, is installed in

\footnotetext{
* Operated by the Univernities Research Association under contract with the U. S. Department of Energy
}

the Fermilab accumulator and has been used to observe escaping ions with various beam conditions.

\section{Detector setup}

The overall system is shown schematically in Fig. 1. The

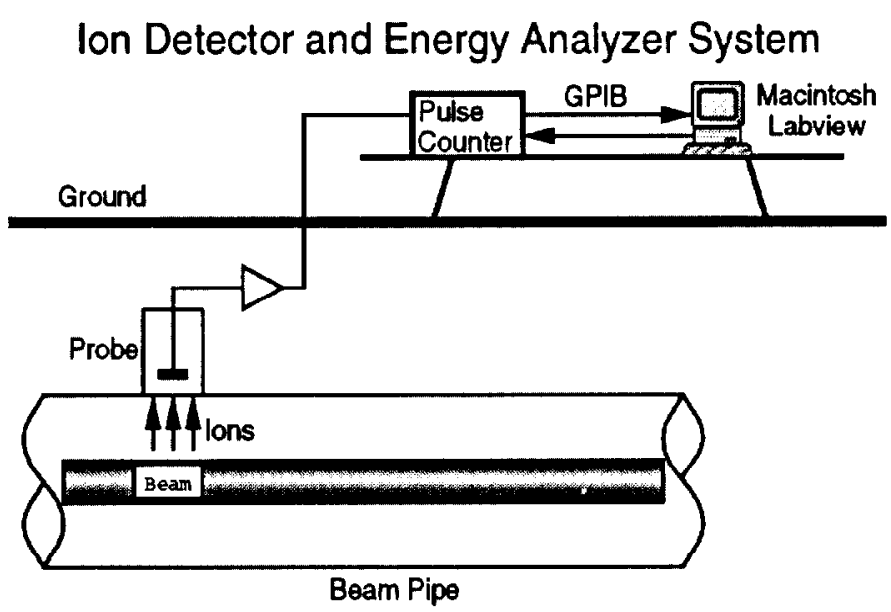

Figure 1: IDEA layout

probe construction is shown in Fig. 2. In the center of the probe is a three stage Micro Channel Plate (MCP) assembly which has a gain of up to a few $10^{7}$ which enables the probe to detect single ions. The front of the MCP assembly is at a negative high voltage, usually $2 k V$ for our operation. This provides the operating voltage for the MCP assembly, but also serves as an acceleration for the ions entering the probe and increases their detection efficiency[2]. Another benefit of this voltage is the exclusion of electrons with energy below $2 \mathrm{keV}$, which should be the majority if not all of the electrons present, from being detected. Voltages can be applied to the plates in front of the MCP to repel lower energy ions, by varying which energy distribution of ions can be measured. The orientation of the probe can be changed nearly $180^{\circ}$. The signal pulses out of the probe, which is mounted in beam pipe vacuum, are then amplified and sent to a pulse counter. Control and count rate measurement are done by a Macintosh computer running Labview ${ }^{\circledR}[3]$. 


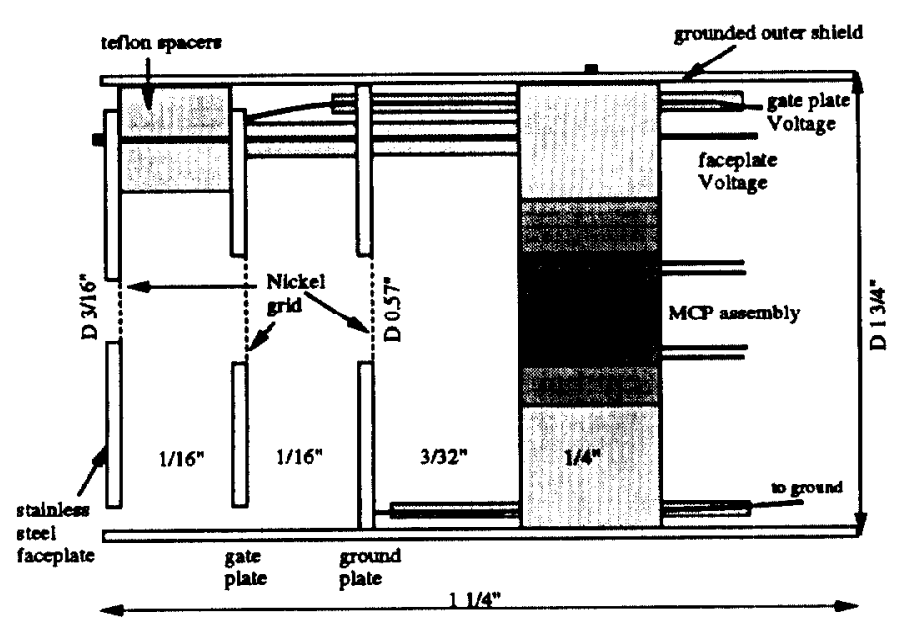

Figure 2: Schematic drawing of IDEA probe

The detector is installed in a drift section, about 1.5 meters to a quadrupole magnet. The lattice functions have the following values, $\beta_{x}=22 \mathrm{~m}, \beta_{y}=2 \mathrm{~m}, D=1.7 \mathrm{~m}$. The pointing angle of the probe is defined such that at 0 it points in the direction of movement of the antiproton beam.

\section{Measurement results}

Angular distributions of escaping ions are measured with both antiproton and proton beams, see Fig. 3 . The accu-

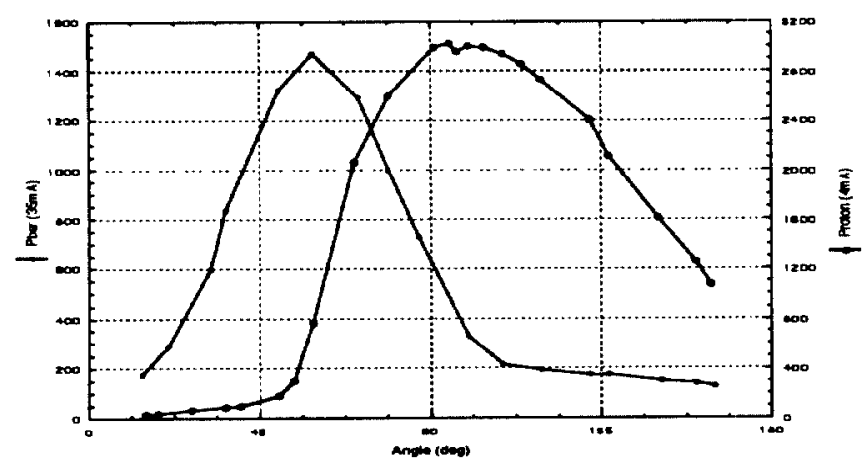

Figure 3: Angular distribution measured with IDEA

mulator sometimes runs with a few mAs of proton beam for the purpose of machine study and tune up. Most of the time proton beams are run in the opposite direction of the antiproton beam, however the proton beam usually has different momentum and therefore passes the detector at a different horizontal position relative to the antiproton beam. Furthermore, protons may have a small crossing angle with the probe because of installation errors, which is estimated at $\pm 5^{\circ}$. The observed difference in angular distribution between proton and antiproton beams is consistent with the hypothesis that horizontally antiproton and proton beams pass the probe on the two sides and a crossing angle causes the probe to face the two beams at different angles and therefore a differently peaked and shaped angular distribution. The crossing angle is within the possible installation tolerance.

The energy distributions measured by IDEA are qualitatively as expected. For the proton beam the electric field of the beam itself pushes positive ions out and therefore should result in a distribution with an mean drift. For the antiproton beam, only ions that have acquired sufficient transverse kinetic energy can escape the beam potential and be detected by our detector. The energy distribution should thus be Maxwellian-like. Fig. 4 shows the comparison of the measured ion energy distribution from a $4 \mathrm{~mA}$ proton beam and one from calculation using a simple model where ions are created without any kinetic energy from an round Gaussian beam. It can be seen from Fig. 4 that they agree reasonably well. Fig. 5 shows the ion energy distribution from a $20 \mathrm{~mA}$ pbar beam. The

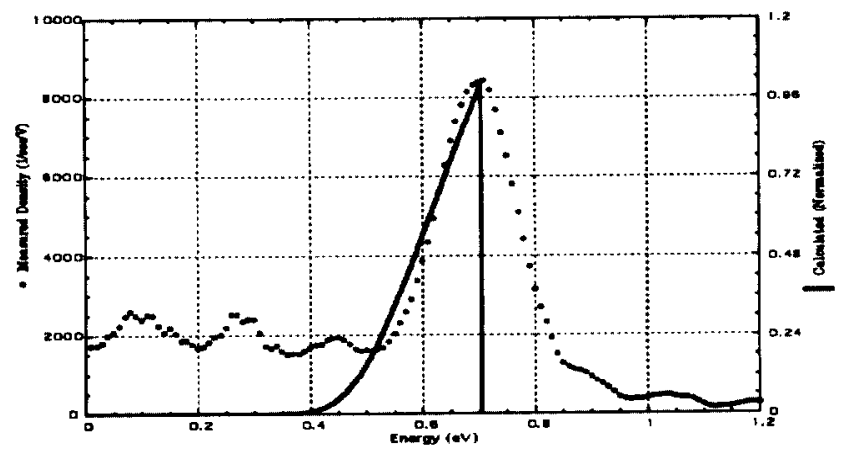

Figure 4: Ion energy distribution from a $4 \mathrm{~mA}$ proton beam

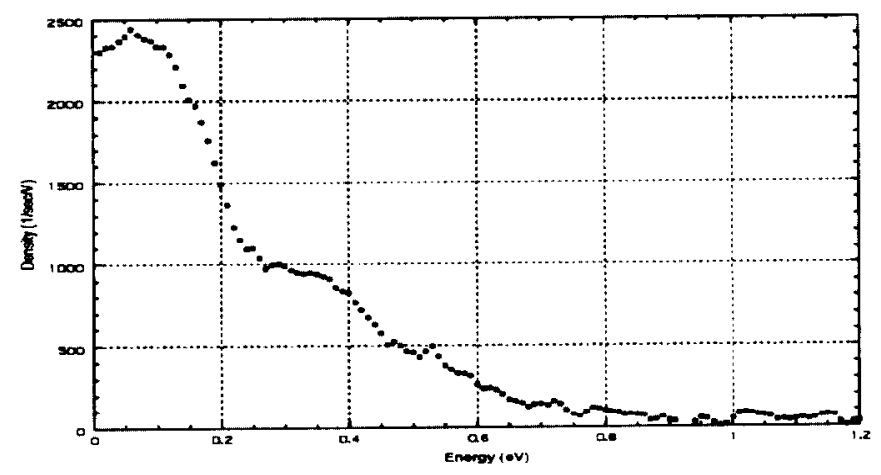

Figure 5: Ion energy distribution from a $20 \mathrm{~mA}$ pbar beam

distribution with antiproton beam shows a very hot ion energy distribution, considering the residual gas temperature. Some ions have energies extending to $1 \mathrm{eV}$ level. This "temperature" seems to be insensitive to the beam current and other conditions of beam. The energy source and transfer mechanism will be discussed later. 
One important motive of making the detector was to eztablish the positive link of beam coherent oscillations and emittance growths with the activity of ions. This is best illustrated as the beam becomes unstable and the emittances undergoes semi-periodic explosive growth. Accompanying the emittance growth there ere bursts of coherent oscillation observed on the coherent pickup. Fig. 6 shows one example. From the figure it can be clearly seen that the large coherent oscillation coincides with the emittance growth and illustrates the cause of that emittance growth. The count rate measured for that period by IDEA, in Fig. 7, shows a very good match to the beam coherent oscillation strength.

One interesting, and still not completely understood, problem is the source of energy of the escaping ions. The usual count rate observed by IDEA is on the order of $10^{3} / \mathrm{sec}$. Electron-ion pairs are created through collision of beam particles, antiprotons in this case, with residual gas molecules. An ion created in this process usually has little kinetic energy $(\lesssim 0.1 \mathrm{eV})$. The electric potential well depth in $\mathrm{eV}$, on the other hand, can be roughly written as $0.2 \times I(m A)$ for typical accumulator conditions. This will mean a $10 \mathrm{eV}$ deep potential well. Therefore, ions have far less initial energy needed to escape the beam trapping field and drift to e.g. IDEA probe. With the detector and beam pipe geometry, the energy flux carried by the flow of ions is estimated to be on the order of $100 \mathrm{GeV} / \mathrm{sec}$. Since residual gas is at room temperature, or "tunnel temperature" to be exact, which at the hottest is about 40$50^{\circ} \mathrm{C}$, ion-gas collisions are, on average, energy depleting interactions for the ions. Other interactions with the environment include charge exchange with neutral molecules or atoms, re-combination with electrons. For accumulator conditions, the former is estimated to have an lifetime of 10-100sec, while the latter has an estimated lifetime orders of magnitudes higher and can thus be ignored. The charge exchange process essentially causes the ion to lose almost all of its kinetic energy to an escaping neutral particle, for which the IDEA probe has a very small detection efficiency[2]. However, a potentially energy gaining interaction for ions is that with the beam. Beam particles have an rms transverse energy of a few $\mathrm{MeV}$, and they have both longitudinal and transverse Schottky noise which can potentially heat the trapped ions relatively fast. A more quantitative investigation of this mechanism is in progress.

\section{Conclusion}

The Ion Detector and Energy Analyzer has been used to successfully detect ions escaping the pbar beam potential well and measure their energy distribution. Ions have unexpectedly high temperatures and their source of energy needs more explanation. ions have been directly observed participating resonantly with the beam coherent oscillations which have led to emittance growth. The observa-

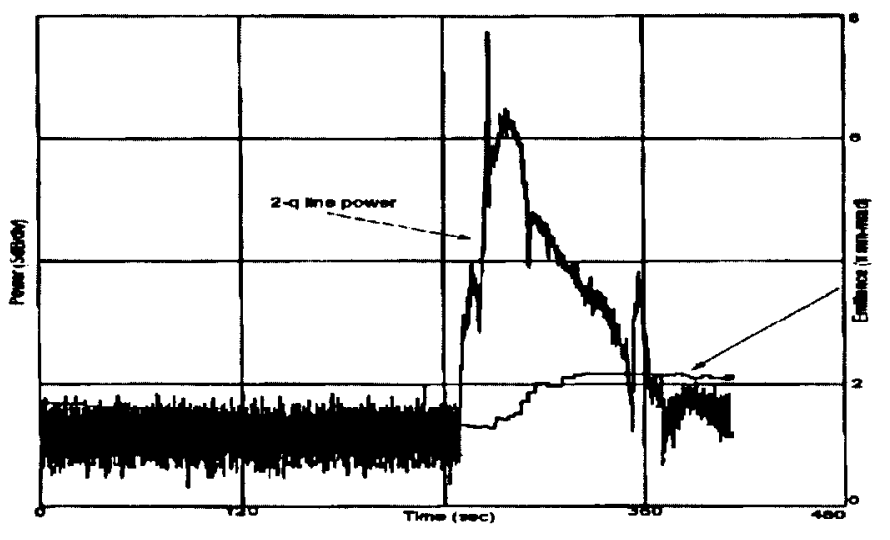

Figure 6: Coherent 2-Q line power at emittance blowup

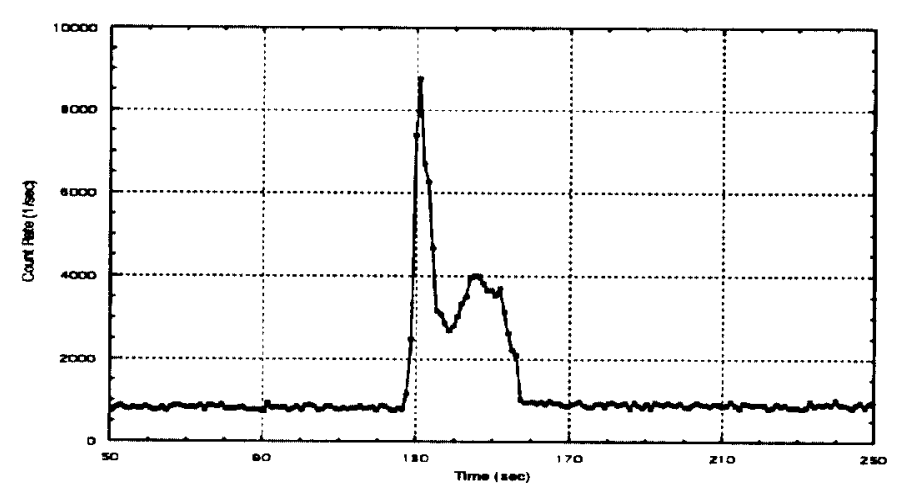

Figure 7: IDEA count rate at emittance blowup

tion of the effect of ions' longitudinal motion is however obstructed by the crossing angle of the detector and the beam.

\section{Acknowledgment}

We would like to thank Dr. Shao Yuan Hsueh for very helpful discussions on energy transfer mechanisms from the bearn to trapped ions. We are also indebted to the Fermilab pbar group for their support and assistance in the installation and setup of IDEA.

\section{References}

[1] Foster F. Rieke and William Prepejchal. Ionization cross sections of gaseous atoms and molecules for highenergy electrons and positrons. Physical Review A, 6(4):1507, October 1972.

[2] Hamamatsu Corporation. Technical Information: MCP Assembly, September 1991.

[3] Labview ${ }^{\circledR}$ is a commercial product and a trademark of National Instruments Corporation. 